A simple calf muscle ergometer for use in a standard whole-body MR scanner.

Quistorff, Bjørn; Nielsen, Steen; Thomsen, Carsten; Jensen, Karl Erik; Henriksen, Ole

Published in:

Magnetic Resonance in Medicine

Publication date:

1990

Document version

Publisher's PDF, also known as Version of record

Citation for published version (APA):

Quistorff, B., Nielsen, S., Thomsen, C., Jensen, K. E., \& Henriksen, O. (1990). A simple calf muscle ergometer for use in a standard whole-body MR scanner. Magnetic Resonance in Medicine, 13(3), 444-449. 


\title{
A Simple Calf Muscle Ergometer for Use in a Standard Whole-Body MR Scanner
}

\author{
Bjørn QuistorfF, ${ }^{*}$ Steen Niel.sen, ${ }^{*}$ Carsten Thomsen, Karl ERik Jensen, \\ AND OLE HENRIKSEN \\ * NMR Center, The Panum Institule, University of Copenhagen, and Department of Magnetic Resonance, \\ Hvidovre Hospital, Copenhagen, Denmark
}

Received November 18, 1988; revised April 3, 1989

\begin{abstract}
A calf muscle ergometer, designed for use in a standard whole-body MR scanner, is described. The instrument allows isotonic or isometric calf muscle cxcrcise aver a continuous rangc of work loads from 0 to individual maximum work output. Work output may be rceordcd on a strip chart reeorder. A sound cncoding provides an effective guidance for the subjeet tested to accurately reproduce his performance in each contraction cycle at a given work load. The FID recording may be triggered from the crgometer at any point during the eontraction cyclc. Nonmetal materials are used for the instrument construction in order to eliminate potentially disturhing eddy currents. The force resistance of the ergometer is obtained by a combined hydraulic-pneumatic system, which is loaded by coupling to an outside source of compressed air equipped with a suitable pressure rcgulator. When varying the lime ol data sampling within a repcated 6-s contraction cycle it was found that the recorded $P_{\mathrm{i}} / \mathrm{PCr}$ ratio remained essentially constant at low and moderate work loads. However, at work loads at or above $70 \%$ of individual $V_{\max }$ a pronounced dependence of the time of sampling with the contraction cycle was observed. (ㄷ) 1990 Academic Press. Inc.
\end{abstract}

\section{INTRODUCTION}

There is a need for noninvasive, quantitative evaluation of the metabolic status in human skeletal muscle during exercise under physiological conditions as well as in the diseased state. The so-called transfer function was recently proposed for this purpose using ${ }^{31} \mathrm{P}$ MRS $(1,2)$, but also ${ }^{13} \mathrm{C}$ MRS is potentially useful for such exercise studies $(3,4)$. This article describes a versatile and inexpensive ergometer designed for use in a standard whole-body MR scanner for routine evaluation of the metabolic function of the human calf muscle. These data were presented in a preliminary form (5).

\section{DESCRIPTION OF THE ERGOMETER}

The ergometer was constructed to be operated in a 55-cm-bore 1.5-T Siemens Magnetom equipped for ${ }^{1} \mathrm{H}$ imaging, 'H MRS, and ${ }^{31} \mathrm{P}$ MRS, Figs. 1 and 2. Nonmetallic materials (PVC and nylon) were used in the construction of the instrument in order to eliminate a potential source of disturbing eddy currents. The ergometer pedal is mounted on a plywood board, $2 \mathrm{~m}$ long, $40 \mathrm{~cm}$ wide, and $1.7 \mathrm{~cm}$ thick, curved to fit the magnet bore. The pedal mount is slightly elevated and allows $6 \mathrm{~cm}$ of lateral 


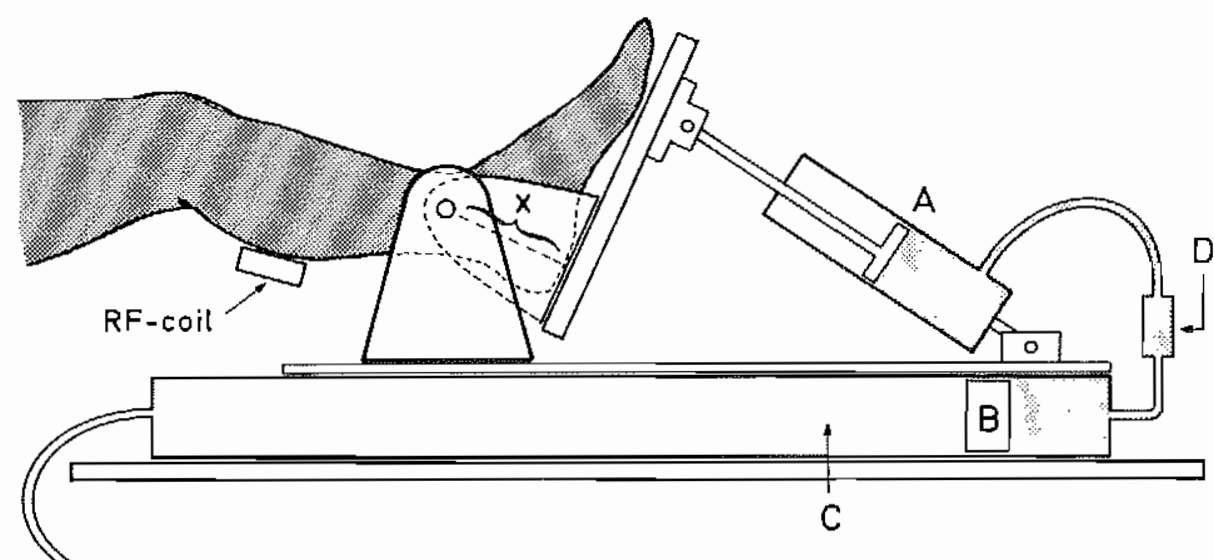

to compressed air cylinder

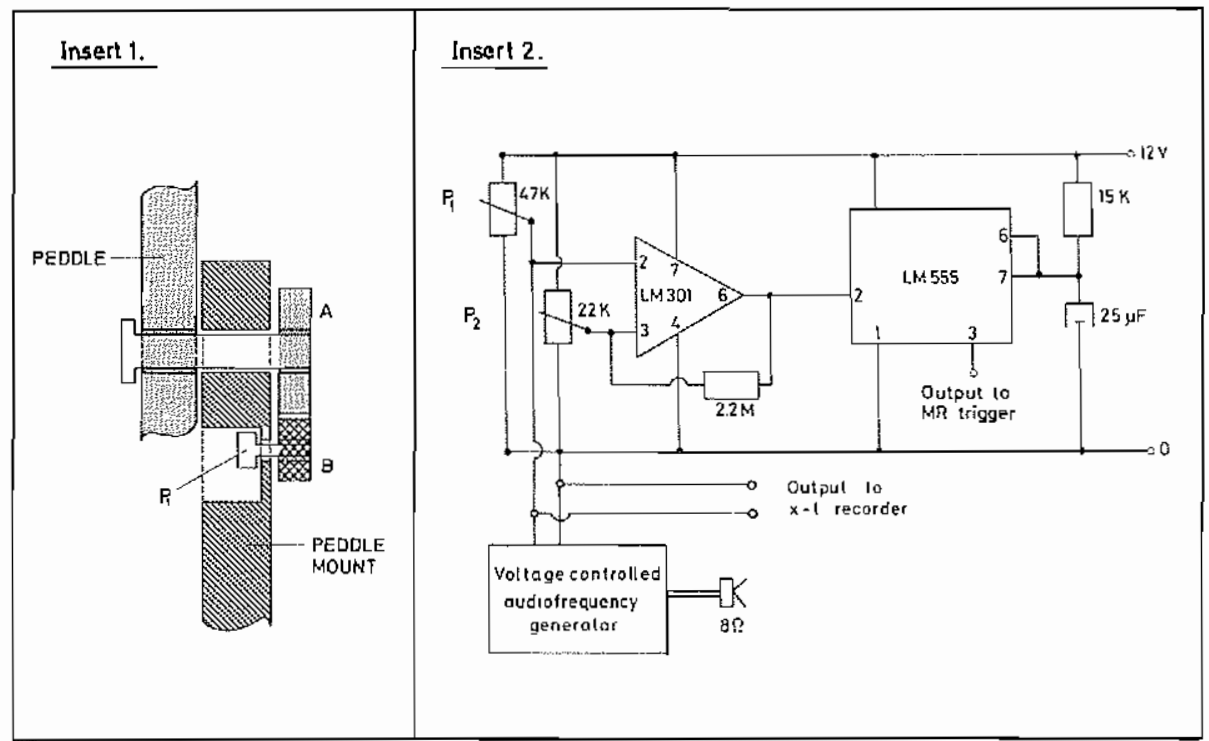

FIG. 1. Schematic diagram of the ergometer. A hydraulic cylinder, $A$, is connected to the ergometer pedal. The mount is adjustable, allowing regulation of the pedal angle at rest, and of the total pedal movement. The cylinder, C, is approximately 30 -fold larger than cylinder A. The freely moving piston, B, separates the pncumatic and the hydraulic (shaded) part of the systcm. D is a choke resistance valve in the hydraulic system controlling the recoil speed of the pedal. Water is used as the hydraulie fluid. The distance, $x$, is adjustable by means of foot plates of varying thiekness. (Insert 1) Detail showing the mount of the angle eneoding $4.7 \mathrm{~K}$ potentiometer, $P_{1}$ which is gcared to the pedal movements via two nylon sprocket wheels, $A$ and $B$, at a ratio of 2:1. (Insert 2) Diagram of the circuit eontrolling the FID triggering, the sound cncoding and output to a strip chart recorder of the pedal movement. Presetting of potentiometer $\mathrm{P}_{2}$ exeeutes the FID triggering signal at the point when the pedal movement has turned the potentiometer $P_{1}$ correspondingly ( see Insert I). 


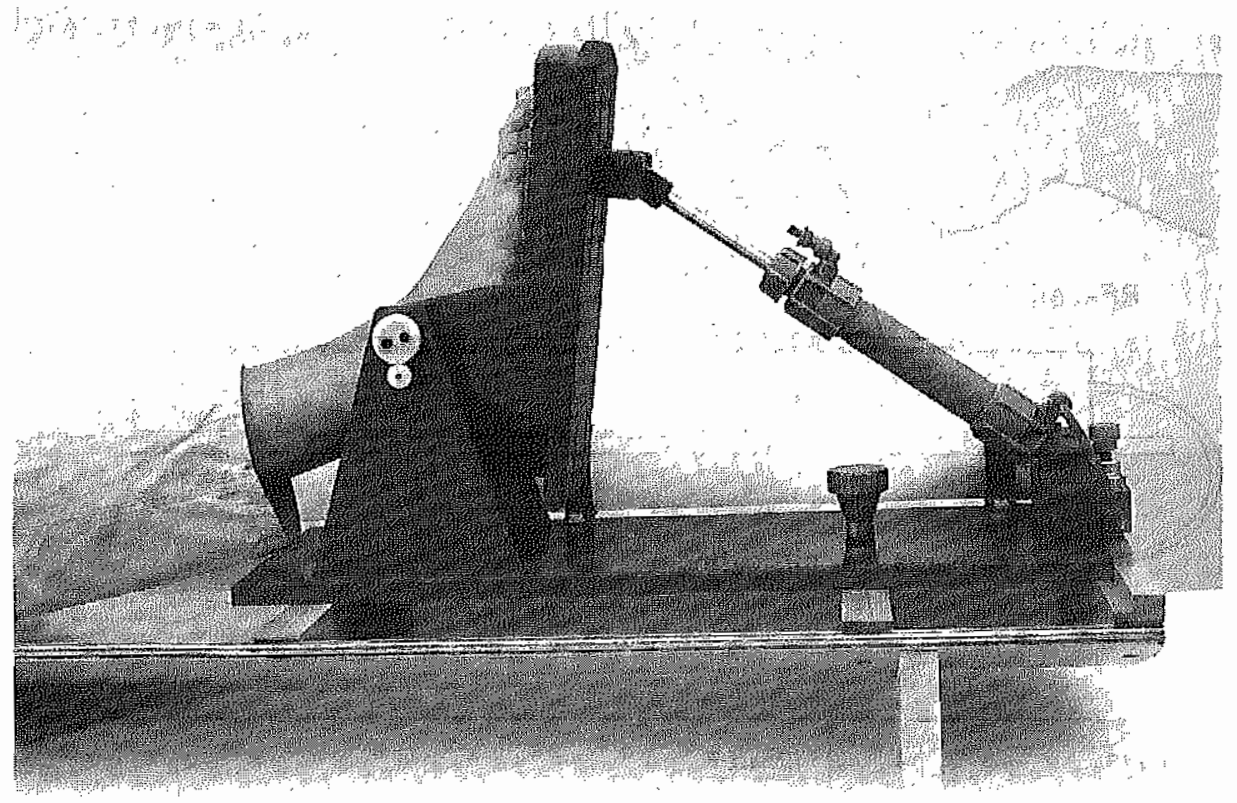

FIG. 2. The ergometer is designed for use in a standard whole-body MR scanner and is constructed with the use of nonmetallic matcrial. For details see tex1 and the legend to Fig. 1.

movement for comfortable use with either leg. Two 5-cm-wide nylon straps support the subject being tested in a fixed position on the board.

The construction of the pedal is such that the axis of the pedal joint can be aligned with the axis of the ankle joint by varying the thickness of the foot plate (a selection of cork plates of different thickncsses that mount on the pedal with a Velcro snap lock). This adjustment possibility is important in order to obtain the correct physiological load on the joint, and in particular in order to keep the calf muscle in a steady position during the NMR recording. The graded force resistance of the pedal is obtained by a combined hydraulic-pneumatic system, as shown schematically in Fig. 1. The piston of a hydraulic cylinder (type DSNK-25-80P/S, Festo Pneumatic, 7300, West Germany) is linked directly to the pedal. Via a one-way choke resistance valve (type LCS-1/4PK6, Festo Pneumatic, 7300, West Germany) this cylinder is connected to a much larger (ca. 30-fold) nylon cylinder mounted alongside the pedal on the board (Fig. 1). A freely moving nylon piston in this latter cylinder separates the hydraulic and the pneumatic system, which again is connected to a compressed air source via an 8-mm-diameter PVC hose. The pressure source is equipped with a 0to $10-\mathrm{kPa}$ pressure regulator allowing the ergometer force resistance to be adjusted continually between 0 and $1250 \mathrm{~N}$. The speed of pedal recoil is adjustable by the choke resistance valve inserted in the hydraulic part of the system between the two cylinders (Fig. 1). A slow recoil of the pedal is important, since otherwise significant work is being performed resisting this movement. Because of the wind-kettle function of the large cylinder, the pressure in the system remains essentially constant (less 
than $3 \%$ change) during the pedal movement. Thus, work may be performed under essentially isotonic conditions. Isometric work is performed and recorded by connecting the hydraulic cylinder, $\mathrm{A}$, directly to a pressure transducer.

During isotonic work the angular movement of the pedal is encoded by a nonmagnetic 4.7-kohm potentiometer geared to the pedal joint (Fig. 1, Insert 1). This potentiometer, $P_{1}$, is part of a simple electrical circuit (Fig. 1 , lnsert 2), which serves three functions: First, in order to standardize the exercise test, it is important that the subject being tested is able to reproduce his performance at a given work load. To achieve that we use a simple acoustic system. The system has proven very efficicnt and works simply by a sound encoding of the angular movement of the pedal, increasing the frequency of the sound as the pedal is pushed down. Sccond, the circuit provides a record of the subject's performance in the form of a DC potential proportional to the linear movement of the piston on the hydraulic cylinder. This signal may be recorded on a strip chart recorder. Third, a trigger signal for the RF pulse is provided. The time of triggcring within the contraction cyclc is continually adjustable by presetting the potentiometer, $\mathrm{P}_{2}$, of the circuit shown in Fig. 1, Insert 2.

The work performed during steady-state exercise with the ergometer may be calculated as the pressurc-movcment product for each contraction and expressed as the average per second, usually of 16 contractions, at each pressurc level. After proper calibration of the pedal movement on the strip chart recorder work may be obtained in watts,

$$
\text { work }=L \times M \times P \times n,
$$

where $L$ is the linear movement (m) of the piston of the hydraulic cylinder (Fig. 1), $M$ is the area of this piston $\left(\mathrm{m}^{2}\right), P$ is the pressure $(\mathrm{Pa})$ of the systcm, and $n$ is the number of contraction cycles per second.

\section{RECORDING OF ${ }^{31} \mathrm{P}$ SPECTRA DURING EXERCISE}

The ergometer was designed in order to obtain near-physiological conditions for the calf muscle exercise; i.e., the knee of the exercising leg is almost fully extended (ca. $170^{\circ}$ ) and the subject is fixed to the board in the longitudinal direction by the two shoulder straps, so that the work performed on the pedal is the result only of the calf muscle activity. The RF coil is strapped on to the medial part of the calf muscle. The coil is a three-turn 8-cm-diameter standard Sicmens surface coil, tuned to the resonance frequency of phosphorus. Each FID is collected in 1024 data points. A dwell time of $250 \mu \mathrm{s}$ ( $2000-\mathrm{Hz}$ spectral bandwidth) and a $500-\mu \mathrm{s}$ delay is applied. After shimming on the proton signal the transmitter power is optimized on the $\mathrm{PCr}$ signal at rest. Under these conditions the "field of view" of the coil includes signal from all three parts of the triceps surae muscle, but mainly from the gastrocnemius.

We standardly apply two different work protocols. One protocol involves a ramp of three to six steady-state work levels between 0 and $70 \%$ of individual $V_{\max }$ with 16 single contractions at each level, essentially as described in (6). $V_{\max }$ is defined as the maximum work level the subject can maintain over a period of $2 \mathrm{~min}$. The single contractions are usually repeated every 5-6 s. With the other protocol work is carried out, usually at about $70 \%$ of individual $V_{\max }$, until exhaustion (inability to push the 


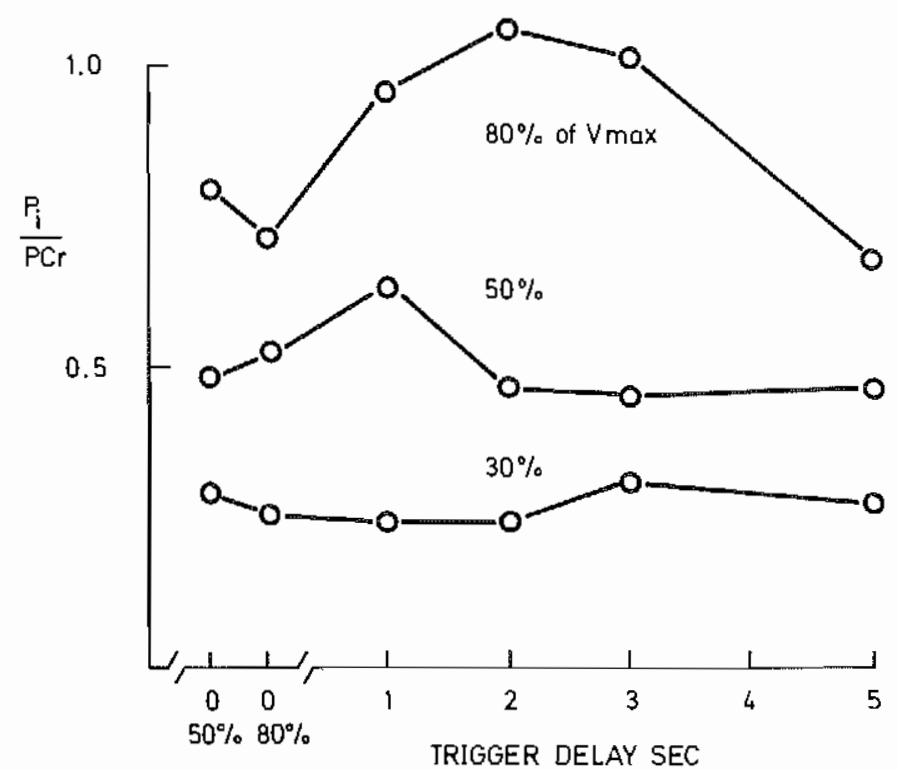

FIG. 3. Relation between trigger delay and the $\mathrm{P}_{\mathrm{i}} / \mathrm{PCr}$ ratio of the ealf muscle of a female volunteer. The experiment was carricd out at three diflerent work levels as indicated on the figure. The points $0_{50 \%}$ and $0_{80 \%}$ refer to FID recordings triggered at the start of the contraction cycle with 50 and $85 \%$ of the active pedal movement completed, respectively.

pedal down). Immediately thereafter the metabolic recovery is followed in spectra obtained every $5 \mathrm{~s}$ for a total of $250 \mathrm{~s}(7)$. Since a single scan using the 8 -cm-diameter surface coil can resolve the five major peaks of the ${ }^{31} \mathrm{P}$ spectrum sufficiently well $(S$ ) $N>10$ ), this protocol in fact affords a 5-s time resolution of the metabolic recovery following exhaustion ( 8 ).

Both protocols may be completed in about an hour including mounting and dismounting of the ergometer in the magnet. Furthermore, shimming is not complicated by the presence of the setup in the magnet. More than 1 year of routine clinical use at the MR department at Hvidovre Hospital in the Siemens Magnetom has demonstrated the ergometer to be simple and reliable to operate ( 8 ).

\section{EFFECT OF VARYING THE TRIGGER POINT WITHIN THE CONTRACTION CYCLE}

The $P_{\mathrm{i}} / \mathrm{PCr}$ ratio has been demonstrated to be a sensitive parameter for energy turnover rate in skeletal muscle, and at low and intermediate work levels there seems to be a linear relation between work output and the $P_{\mathrm{i}} / \mathrm{PCr}$ ratio $(1,2)$. Since the $\dot{V} \mathrm{O}_{2}$ is also linearly related to work output under steady-state conditions $(9)$ the $P_{\mathrm{i}} /$ $\mathrm{PCr}$ ratio can be assumed to be proportional to the $\dot{V} \mathrm{O}_{2}$ of the muscle under such conditions of steady state. We have adopted the $P_{\mathrm{i}} / \mathrm{PCr}$ parameter in our standard work protocol, and in order to define optimal test conditions we have systematically tested the effect on the $P_{\mathrm{i}} / \mathrm{PCr}$ ratio of varying the trigger point for the RF pulse within the contraction cycle. 
The experiments were carried out at three steady-state work levels, at 30,50, and $70 \%$ of individual $V_{\max }$. At each work level six separate experiments were carried out with 16 contractions in each experiment and $6 \mathrm{~s}$ between contractions. FIDs were collected with a 0 -s delay in the first experiment, with a $1-\mathrm{s}$ delay in the next, etc., and a 5-s delay in the last. In other words, each of the three graphs in Fig. 3 represents six individual experiments, which are identical, except for the fact that the FID sampling was performed at different points in the contraction cycle. With a 0 -s delay two sets of data were collected, one with the FID trigger point set corresponding to $50 \%$ of maximum contraction and the other at $85 \%$ contraction, i.e., just before contraction maximum. Thus, the experiment was designed to show the metabolic relaxation pattern in between contractions at different work levels, as judged by the $P_{\mathrm{i}} / \mathrm{PCr}$ ratio.

Four volunteers (two males and two females) were tested. A typical result is shown in Fig. 3. Below work levels of 40-50\% of individual $V_{\max }$ the $P_{\mathrm{i}} / \mathrm{PCr}$ ratio was essentially independent of the trigger point. However, at highcr work levels $(70 \%$ and above) a nonlinear dependence of the time of triggering of the FID within the contraction cycle was observed. In the example shown in Fig. 3 the $P_{\mathrm{i}} / \mathrm{PCr}$ ratio was significantly increased at $1-$ to 3 -s delays compared with the value observed at the 0 and 5-s delays. In other words, the linearity between work output and the $P_{\mathrm{i}} / \mathrm{PCr}$ ratio seems to depend upon the point of measurement within the contraction cycle. This unexpected recovery pattern within the contraction cycle at high work loads may be related to rccruitment of glycolytic fibers in the muscle. In fact, we have in some of these experiments observed a splitting of the $P_{\mathrm{i}}$ peak, (corresponding to 0.35 $0.51 \mathrm{pH}$ units) which speaks in favor of such an explanation as also observed by Park et al. $(10)$.

\section{ACKNOWLEDGMENTS}

This work was supported by Statens Liegevidenskabelige Forskningsrăd, J.nr. FTU 5175214 and The NOVO Foundation, J.nr. 85-12-11.

\section{REFERENCES}

1. B. Chance, J. S. Leigh, B. J. Clark, J. Maris, J. Kent, S. Nioka, and D. Smith, Proc. Natl. Acad. Sci. USA 82,8384 (1985).

2. B. Chance, J. S. Litigh, J. Kent, K. MoCully, S. Nioka, B. J. Clark, J. M. Maris, and T. GRAHAM, Proc. Nall. Acad. Sci. USA 86, 9458 (1986).

3. B. QuistorfF, S. Wicklund, J. S. Leigh, AND B. ChanCE, "7th Ann. Meet. Soc. Magn. Res.," Vol. 1, Abstraet 312, 1988.

4. T. Jue, J. Lohman, R. J. Ordidge, and R. G. Shulman, Magn. Reson. Med. 5, 377 (1987).

5. B. Quistorf, K. E. Jensen, O. Henriksen, And C. Thomsen, "7th Ann. Meet. Soc. Magn. Res.," Vol. 3, Abstract 95, 1988.

6. K. MCCUl.LX, J. Kent, AND B. ChanCe, Sports Med. 5, 312 (1988),

7. D. J. Taylor, P. Styles, P. M. Matthews, D. A. ARnold, D. G. Gadian, P. Bore, and G. K. RadDA, Magn. Reson. Med. 3, (1986).

8. J. Jakobsen, C. ThOMSEN, C-E. Jensen, AND O. HenRIKSEN, submilled for publication.

9. B. SALtin, Amer, J. Cardiol. 55, 42D (1985).

10. J. H. Park, R. L. Brown, C. R. Park, K. MoCully, M. Cohen, J. Haselgrove, and B. Chance, Proc. Nall. Acad. Sci. USA 84, 8976(1987). 